\title{
Single-qubit lasing and cooling at the Rabi frequency
}

\author{
Julian Hauss ${ }^{1,2}$, Arkady Fedorov ${ }^{1}$, Carsten Hutter ${ }^{1,3}$, Alexander Shnirman ${ }^{1,4}$, and Gerd Schön ${ }^{1}$ \\ ${ }^{1}$ Institut für Theoretische Festkörperphysik and DFG-Center for Functional Nanostructures (CFN), \\ Universität Karlsruhe, D-76128 Karlsruhe, Germany \\ 2 Lichttechnisches Institut, Universität Karlsruhe, D-76128 Karlsruhe, Germany \\ ${ }^{3}$ Department of Physics, Stockholm University, AlbaNova University Center, SE - 10691 Stockholm, Sweden and \\ 4 Institut für Theoretische Physik, Universität Innsbruck, A-6020 Innsbruck, Austria
}

\begin{abstract}
For a superconducting qubit driven to perform Rabi oscillations and coupled to a slow electromagnetic or nano-mechanical oscillator we describe previously unexplored quantum optics effects. When the Rabi frequency is tuned to resonance with the oscillator the latter can be driven far from equilibrium. Blue detuned driving leads to a population inversion in the qubit and a bi-stability with lasing behavior of the oscillator; for red detuning the qubit cools the oscillator. This behavior persists at the symmetry point where the qubit-oscillator coupling is quadratic and decoherence effects are minimized. There the system realizes a "single-atom-two-photon laser".
\end{abstract}

Several recent experiments on quantum state engineering with superconducting circuits [1, 2, 3] realized concepts originally introduced in the field of quantum optics and stimulated substantial theoretical activities [4, 5]. Josephson qubits play the role of two-level atoms, while oscillators of various kinds replace the quantized light field. Motivated by one such experiment [1], we investigate a Josephson qubit coupled to a slow LC oscillator (Fig. 1 a) with eigenfrequency (in the $\mathrm{MHz}$ range) much lower than the qubit's energy splitting (in the GHz range), $\omega_{T} \ll \Delta E$. The qubit is ac-driven to perform Rabi oscillations, and the Rabi frequency $\Omega_{R}$ is tuned close to resonance with the oscillator. For this previously unexplored regime of frequencies we study both onephoton (for $\Omega_{R} \approx \omega_{T}$ ) and two-photon (for $\Omega_{R} \approx 2 \omega_{T}$ ) qubit-oscillator couplings. The latter is dominant at the "sweet" point of the qubit, where due to symmetry the linear coupling to the noise sources is tuned to zero and dephasing effects are minimized [6]. When the qubit driving frequency is blue detuned, $\delta \omega=\omega_{d}-\Delta E>0$, we find that the system exhibits lasing behavior; for red detuning the qubit cools the oscillator. Similar behavior is expected in an accessible range of parameters for a Josephson qubit coupled to a nano-mechanical oscillator (Fig. 1b), thus providing a realization of a SASER 7] (Sound Amplifier by Stimulated Emission of Radiation).

Our work is motivated by a recent experiment [1] where under the (assumed) condition $\Omega_{R} \approx \omega_{T}$ a strong excitation of the LC oscillator was observed. Previous attempts to explain this behavior [8, 9] did not resolve several issues: First, according to these theories the effect should vanish at the symmetry point, but it was observed there as well. A possible explanation [1, 8], assuming uncontrolled small deviations from the symmetry point, is not supported by experiments. Here we explore an alternative, namely that 2 -photon processes at $\Omega_{R} \approx 2 \omega_{T}$ which persist at the symmetry point, are responsible for the observed effect.

The second unresolved problem is the magnitude of the effect. The experiment showed an increase by a factor

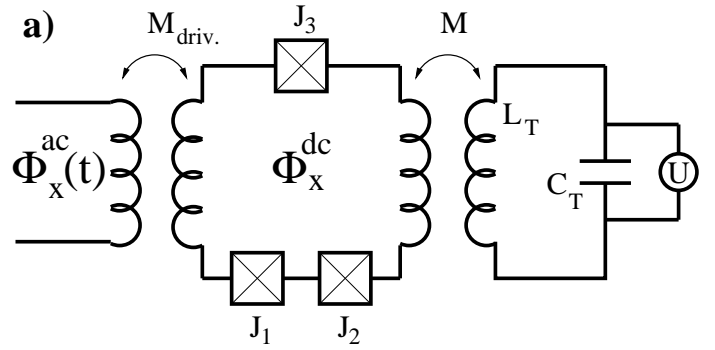

b)

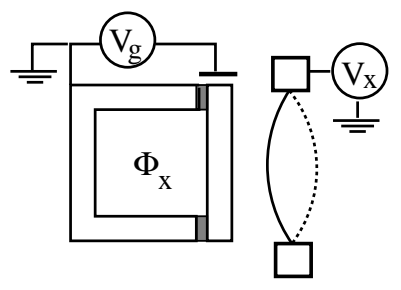

FIG. 1: The systems. a) In the circuit QED setup of Ref. [1] an externally driven three-junction flux qubit is coupled inductively to an LC oscillator. b) In an equivalent setup a charge qubit is coupled to a mechanical resonator.

$\sim 10$ in the number of oscillator quanta [1]. The theory of Ref. 8], valid in the perturbative regime, predicts a much weaker effect. Here we demonstrate that the highfrequency driving of the qubit, in combination with the unusual resonance condition introduces qualitatively new effects: Namely, for blue detuning of the qubit driving a population inversion is created between the qubit dressed states, which are split by the Rabi frequency [10, 11]. Under these conditions the ac-driving provides more energy than needed to excite the qubit, and excess energy is flowing into the resonator. The lasing threshold and a proper lasing state with the characteristic line narrowing can be reached, and the system becomes a "single-atomlaser" [12]. Similarly, for red detuning we predict strong cooling of the oscillator.

The described mechanism is strong enough to explain the observed level of excitation of the oscillator. At this 
stage, using parameters provided by Ref. [1], we have not reached quantitative agreement with experiment, possibly because of a combination of strong drive and low quality factor. However, the effects should be observable in circuit QED setups of the type described if sufficiently high $Q$-factor can be achieved. Furthermore, our analysis applies for low-frequency nano-mechanical resonators, where sufficiently high $Q$ - factor have already been obtained, and true lasing (sasing) should arise.

The systems considered are shown in Fig. 1. To be specific we first analyze the Rabi driven flux qubit coupled to an LC-oscillator (Fig. 1 $\mathrm{k}$ ) with Hamiltonian

$$
\begin{aligned}
H= & -\frac{1}{2} \epsilon\left(\Phi_{x}^{d c}\right) \sigma_{z}-\frac{1}{2} \Delta \sigma_{x}-\Omega_{R 0} \cos \left(\omega_{d} t\right) \sigma_{z} \\
& +\omega_{T} a^{\dagger} a+g \sigma_{z}\left(a+a^{\dagger}\right) .
\end{aligned}
$$

The first two terms describe the qubit, with Pauli matrices acting on the flux basis states of the qubit. The energy bias $\epsilon\left(\Phi_{x}^{d c}\right)$ between the flux states is controlled by a dc magnetic flux, and $\Delta$ is the tunneling amplitude between both. The third term accounts for the driving of the qubit by an applied ac flux with amplitude $\Omega_{R 0}$ and frequency $\omega_{d}$. The last two terms describe the oscillator with frequency $\omega_{T}=\left(L_{T} C_{T}\right)^{-1 / 2}$, as well as the qubitoscillator interaction. We estimate the coupling constant as $g \approx M I_{p} I_{T, 0}$, where $M$ is the mutual inductance, $I_{p}$ the magnitude of the persistent current in the qubit, and $I_{T, 0}=\left(\omega_{T} / 2 L_{T}\right)^{1 / 2}$ the amplitude of the vacuum fluctuation of the current in the LC-oscillator.

After transformation to the eigenbasis of the qubit, a Schrieffer-Wolff transformation, and a rotating wave approximation (RWA) the Hamiltonian reads

$$
\begin{aligned}
H & =-\frac{1}{2} \Delta E \sigma_{z}+\Omega_{R 0} \cos \left(\omega_{d} t\right) \cos \zeta \sigma_{x}+\omega_{T} a^{\dagger} a \\
& +g \sin \zeta \sigma_{z}\left(a+a^{\dagger}\right)-\frac{g^{2}}{\Delta E} \cos ^{2} \zeta \sigma_{z}\left(a+a^{\dagger}\right)^{2}
\end{aligned}
$$

where $\Delta E \equiv \sqrt{\epsilon^{2}+\Delta^{2}}$ and $\tan \zeta=\epsilon / \Delta$. The derivation resembles that of Ref. [5] in the dispersive regime, except that we keep terms proportional to $a^{2}$ and $a^{\dagger^{2}}$. They cannot be dropped, since the oscillator's frequency, $\omega_{T}$, is much lower than $\Delta E$. Hence, $a^{2}$ and $a^{\dagger^{2}}$ evolve slowly.

Next we transform the Hamiltonian (2) into the rotating frame, which introduces the full Rabi frequency $\Omega_{R}=$ $\sqrt{\Omega_{R 0}^{2} \cos ^{2} \zeta+\delta \omega^{2}}$, and an angle $\tan \beta=\delta \omega /\left(\Omega_{R 0} \cos \zeta\right)$ characterizing the detuning $\delta \omega \equiv \omega_{d}-\Delta E$. We employ a second RWA in which we consider $\omega_{T}$ and $\Omega_{R} \sim \omega_{T}$ as fast (see justification below). In the interaction representation with respect to the non-interacting Hamiltonian $H_{0}=\left(\Omega_{R} / 2\right) \sigma_{z}+\omega_{T} a^{\dagger} a$, we arrive at

$$
\begin{aligned}
H_{I}^{R} & =g_{1}\left(a^{\dagger} \sigma_{-} e^{-i\left(\Omega_{R}-\omega_{T}\right) t}+\text { h.c. }\right) \\
& +g_{2}\left(a^{\dagger 2} \sigma_{-} e^{-i\left(\Omega_{R}-2 \omega_{T}\right) t}+\text { h.c. }\right) \\
& +g_{3}\left(a^{\dagger} a+a a^{\dagger}\right) \sigma_{z} .
\end{aligned}
$$

We kept both single- and two-photon interactions with $g_{1}=g \sin \zeta \cos \beta$ and $g_{2}=\left(g^{2} / \Delta\right) \cos ^{2} \zeta \cos \beta$, although within the RWA only one of them survives: the first one near the resonance at $\Omega_{R} \sim \omega_{T}$, the second one for $\Omega_{R} \sim$ $2 \omega_{T}$. The third term with $g_{3}=-\left(g^{2} / \Delta\right) \cos ^{2} \zeta \sin \beta$ causes a frequency shift of the oscillator [13]. All three coupling constants are much smaller than the bare $g$ in the vicinity of the symmetry point. In what follows we assume that the qubit is kept near the symmetry point, i.e., $\epsilon \ll \Delta$ and $\cos \zeta \simeq 1$. Exactly at the symmetry point the single-photon coupling constant $g_{1}$ vanishes, but the two-photon couplings $g_{2}$ and $g_{3}$ persist.

To account for the effects of dissipation we introduce two damping terms in the Liouville equation for the density operator of the system, $\dot{\rho}=$ $-i[H, \rho]+L_{Q} \rho+L_{R} \rho$. As far as the qubit is concerned we consider only spontaneous emission with rate $\Gamma_{0}$. Hence $L_{Q} \rho=\Gamma_{0}\left(2 \sigma_{-} \rho \sigma_{+}-\rho \sigma_{+} \sigma_{-}-\sigma_{+} \sigma_{-} \rho\right) / 2$. This restriction is justified as long as the temperature is lower than the qubit's energy splitting, and the system is biased near the degeneracy point $(\epsilon=$ 0 ) where the additional "pure" dephasing is weak. The resonator damping can be expressed in standard form [14], $L_{R} \rho=\kappa\left(N_{\mathrm{th}}+1\right)\left(2 a \rho a^{\dagger}-a^{\dagger} a \rho-\rho a^{\dagger} a\right) / 2+$ $\kappa N_{\text {th }}\left(2 a^{\dagger} \rho a-a a^{\dagger} \rho-\rho a a^{\dagger}\right) / 2$, where $\kappa$ denotes the resonator width and $N_{\mathrm{th}}=1 /\left[\exp \left(\omega_{T} / k_{B} T\right)-1\right]$ the thermal average number of photons in the resonator. Since we consider qubits with high degree of coherence, we are motivated to study the regime $\Gamma_{0}, \kappa, g_{1}, g_{2}, g_{3} \ll \omega_{T}, \Omega_{R}$, where the second RWA performed above with respect to frequencies $\omega_{T}, \Omega_{R}$ is justified.

After transformations to the rotating frame and the interaction representation, within the RWA the qubit damping takes the form

$$
\begin{aligned}
L_{Q}^{R} \rho_{I}^{R} & =\frac{\Gamma_{\downarrow}}{2}\left(2 \sigma_{-} \rho_{I}^{R} \sigma_{+}-\rho_{I}^{R} \sigma_{+} \sigma_{-}-\sigma_{+} \sigma_{-} \rho_{I}^{R}\right) \\
& +\frac{\Gamma_{\uparrow}}{2}\left(2 \sigma_{+} \rho_{I}^{R} \sigma_{-}-\rho_{I}^{R} \sigma_{-} \sigma_{+}-\sigma_{-} \sigma_{+} \rho_{I}^{R}\right) \\
& +\frac{\Gamma_{\varphi}^{*}}{2}\left(\sigma_{z} \rho_{I}^{R} \sigma_{z}-\rho_{I}^{R}\right) .
\end{aligned}
$$

Remarkably, although we started from spontaneous emission only, the transformation to the rotating frame introduces transition in either direction with rates $\Gamma_{\uparrow, \downarrow}=$ $\frac{\Gamma_{0}}{4}(1 \pm \sin \beta)^{2}$ and an additional pure dephasing term, $\Gamma_{\varphi}^{*}=\frac{\Gamma_{0}}{2} \cos ^{2} \beta$. Right on resonance, where $\beta=0$, we have $\Gamma_{\uparrow}=\Gamma_{\downarrow}$, corresponding to infinite temperature. We further note that detuning modifies the effective temperature in the rotating frame. For blue detuning, $\beta>0$, we find $\Gamma_{\uparrow}>\Gamma_{\downarrow}$, i.e., negative temperature.

The Hamiltonian (3) combined with the Liouville equation and qubit damping term (4) provides the the theoretical description of the system. It differs from earlier work in the unusual resonance condition with the Rabi frequency and the inclusion of both 1- and 2-photon processes. The latter dominate at 
the symmetry point, where decoherence effects are minimized. Below we analyze both resonance situations $\Omega_{R} \approx \omega_{T}$ or $\Omega_{R} \approx 2 \omega_{T}$, and investigate the effects of blue or red detuning, $\delta \omega=\omega_{d}-\Delta E$, of the qubit driving frequency. We proceed in the frame of the standard semi-classical approach [14, 15] of laser physics.

(i) One-photon interaction. When the Rabi frequency is near the resonance with the oscillator, $\Omega_{R} \approx \omega_{T}$, we can neglect in RWA in the Hamiltonian (3) the term with coupling constant $g_{2}$. If we - to start with - neglect fluctuations, the system is described by Maxwell-Bloch equations for the classical variables $\alpha=\langle a\rangle, \alpha^{*}=\left\langle a^{\dagger}\right\rangle$, $s_{ \pm}=\left\langle\sigma_{ \pm}\right\rangle$, and $s_{z}=\left\langle\sigma_{z}\right\rangle$, which can be derived from the Hamiltonian if all correlation functions are assumed to factorize. The qubit variables can be adiabatically eliminated as long as $\kappa, g_{1} \ll \Gamma_{0}$, which leads to a closed equation of motion for $\alpha$. If we account for fluctuations, e.g., due to thermal noise in the resonator, $\alpha$ becomes a stochastic variable obeying a Langevin equation [14],

$$
\dot{\alpha}=-\left[\kappa-\frac{C}{\Gamma_{\varphi}+i \delta \Omega} s_{z}^{s t}+4 i g_{3} s_{z}^{s t}\right] \frac{\alpha}{2}+\xi(t) .
$$

Here $C \equiv 2 g_{1}^{2}, s_{z}^{s t}=-D_{0} /\left(1+\left|\alpha^{2}\right| / \tilde{n}_{0}\right)$ is the stationary value of the population difference between the qubit levels, and $D_{0}=\left(\Gamma_{\downarrow}-\Gamma_{\uparrow}\right) / \Gamma_{1}$ is the normalized difference between the rates with $\Gamma_{1}=\Gamma_{\uparrow}+\Gamma_{\downarrow}$. We further introduced the photon saturation number $n_{0}=\Gamma_{\varphi} \Gamma_{1} / 4 g_{1}^{2}$ and $\tilde{n}_{0} \equiv n_{0}\left(1+\delta \Omega^{2} / \Gamma_{\varphi}^{2}\right)$, and the total dephasing rate $\Gamma_{\varphi}=\Gamma_{1} / 2+\Gamma_{\varphi}^{*}$. The detuning of the Rabi frequency enters in combination with a frequency renormalization, $\delta \Omega \equiv \Omega_{R}-\omega_{T}+4 g_{3}|\alpha|^{2}$. The Langevin force due to thermal noise in the oscillator satisfies $\left\langle\xi(t) \xi^{*}\left(t^{\prime}\right)\right\rangle=\kappa N_{\mathrm{th}} \delta\left(t-t^{\prime}\right)$ and $\left\langle\xi(t) \xi\left(t^{\prime}\right)\right\rangle=0$. Noise originating from the qubit can be neglected provided the thermal noise is strong, $\kappa N_{\text {th }} \gg g_{1}^{2} / \Gamma_{\varphi}$.

(ii) Two-photon interaction. The two-photon effect dominates near the resonance condition $\Omega_{R} \approx 2 \omega_{T}$. In this case in RWA we neglect in (3) the term with coupling constant $g_{1}$. The corresponding Langevin equation for the resonator variable is of the same form as Eq. (5) but with $C \equiv 4 g_{2}^{2}|\alpha|^{2}$ and $s_{z}^{s t}=-D_{0} /\left(1+\left(\left|\alpha^{2}\right| / \tilde{n}_{0}\right)^{2}\right)$. The photon saturation number is now given by $n_{0}=$ $\left(\Gamma_{\varphi} \Gamma_{1} / 4 g_{2}^{2}\right)^{1 / 2}$, and $\tilde{n}_{0} \equiv n_{0}\left(1+\delta \Omega^{2} / \Gamma_{\varphi}^{2}\right)^{1 / 2}$. Again $\xi(t)$ represents thermal noise, while noise arising from the qubit can be neglected if $\kappa N_{\text {th }} \gg g_{2}^{2} \bar{n} / \Gamma_{\varphi}$, where $\bar{n} \equiv\left\langle|\alpha|^{2}\right\rangle$ is the average number of photons. The relevant detuning of the Rabi frequency for two-photon interaction is given by $\delta \Omega \equiv \Omega_{R}-2 \omega_{T}+4 g_{3}|\alpha|^{2}$.

In Fig. 2 we summarize our main results obtained by solving the Langevin (Fokker-Plank) equations [15]. The number of photons $\bar{n}$ is plotted as a function of the detuning $\delta \omega$ of the driving frequency and driving amplitude $\Omega_{R 0}$. It exhibits sharp extrema along two curves corresponding to the one- and two-photon resonances,

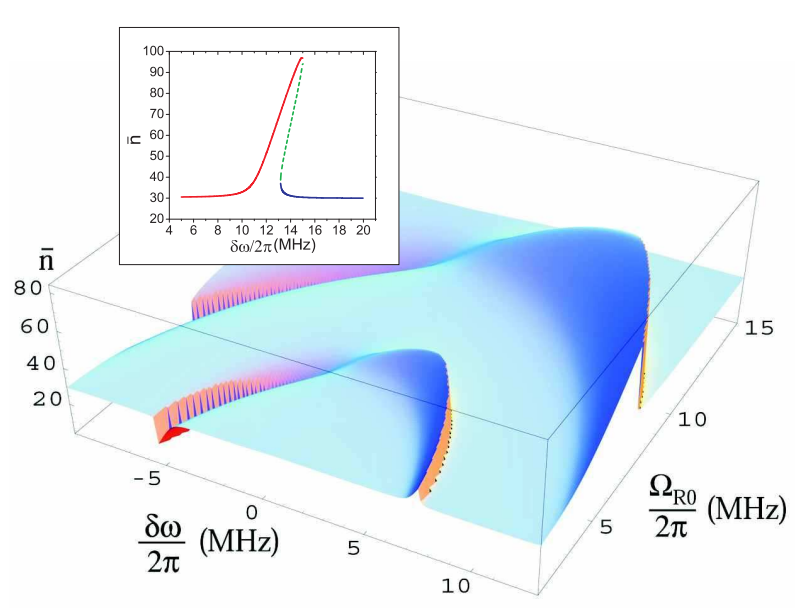

FIG. 2: Average number of photons in the resonator as function of the driving detuning $\delta \omega$ and amplitude $\Omega_{R 0}$. Peaks at $\delta \omega>0$ correspond to lasing, dips at $\delta \omega<0$ to cooling. The inner curve is the one-photon resonance, which exists only away from the symmetry point. (Here we assume $\epsilon=0.01 \Delta$.) The outer curve is the two-photon resonance, which persists at $\epsilon=0$. In domains of bi-stability the lowest value of $\bar{n}$ is plotted. Sharp drops in the curves are related to the bi-stability bifurcations. The parameters for the qubit are $\Delta / 2 \pi=1 \mathrm{GHz}, \epsilon=0.01 \Delta$, and $\Gamma_{0} / 2 \pi=125 \mathrm{kHz}$, the frequency and line-width of the resonator are $\omega_{T} / 2 \pi=6 \mathrm{MHz}$ and $\kappa / 2 \pi=1.7 \mathrm{kHz}$, the coupling constant is $g / 2 \pi=3.3 \mathrm{MHz}$ and the temperature of the resonator $T=10 \mathrm{mK}$. The inset shows the bistability of the photon number for $\Omega_{R 0} / 2 \pi=7 \mathrm{MHz}$. The dashed line represents the unstable solution.

$\Omega_{R}=\omega_{T}-4 g_{3} \bar{n}$ and $\Omega_{R}=2 \omega_{T}-4 g_{3} \bar{n}$. Blue detuning, $\delta \omega>0$, induces a strong population inversion of the qubit levels, which in resonance leads to one-qubit lasing. In experiments the effect can be measured as a strong increase of the photon number in the resonator above the thermal values. On the other hand, red detuning produces a one-qubit cooler with photon numbers substantially below the thermal value. Near the resonances we find regions of bi-stability illustrated in the inset of Fig. 2. In these regions we expect a telegraph-like noise due to random switching between the two solutions.

For strong population inversion, $\Gamma_{\downarrow} \ll \Gamma_{\uparrow}$, i.e., $D_{0} \sim$ -1 , we obtain $\bar{n} \sim N_{\text {th }}+\Gamma_{1} /(2 \kappa)$ for single-photon and $\bar{n} \sim N_{\text {th }}+\Gamma_{1} / \kappa$ for two-photon resonances. If $\Gamma_{1} / \kappa \gg N_{\text {th }}$ we obtain the usual lasing state with Poisson distribution $p_{n}$ and corresponding line narrowing. In the opposite limit the state is almost thermal.

For comparison we plot our results using parameters as provided in Ref. 1]. As shown in Fig. 2 blue detuning causes a increase in the resonator occupation number by a factor $\sim 3$, a value which is short of the experimentally observed one. By adjusting the parameters we can reach enhancements comparable to the observed ones. It requires either decreasing $\kappa$ by a factor of $\sim 4$ or increasing $\Gamma_{0}$ by a factor of $\sim 4$ or a combination thereof. Further 
analysis and fitting as well as studies of higher- $Q$ circuits should help resolving the remaining discrepancies.

So far we described a flux qubit coupled to an LC oscillator, but our analysis applies equally to a nanomechanical resonator capacitively coupled to a Josephson charge qubit (see Fig. 1b). In this case $\sigma_{z}$ stands for the charge of the qubit, and both the coupling to the oscillator and the driving are capacitive, i.e., involve $\sigma_{z}$. To produce capacitive coupling between the qubit and the oscillator, the latter is metal coated and charged by a voltage source [3]. The dc component of the gate voltage $V_{g}$ puts the system near the charge degeneracy point where the dephasing due to the $1 / f$ charge noise is minimal. Rabi driving is induced by an ac component of $V_{g}$. Realistic experimental parameters are expected to be very similar to the ones used in the examples discussed above, except that a much higher quality factor of the resonator $\left(\sim 10^{5}\right)$ and a much higher number of quanta in the oscillator can be reached. This number will easily exceed the thermal one, thus a proper lasing state with Poisson statistics, appropriately named SASER [7], is produced. One should then observe the usual line narrowing with line width given by $\kappa N_{\text {th }} /(4 \bar{n}) \sim \kappa^{2} N_{\text {th }} / \Gamma_{1}$. Experimental observation of this line-width narrowing would constitute a confirmation of the lasing/sasing.

Another useful application of the considered scheme is the cooling of the resonator. From our analysis we conclude that a population of order $\bar{n}=1$ can be reached for optimal detuning. Further analysis is required to determine the optimum conditions.

The effect which we described here differs from the "dressed-state lasing" studied earlier in quantum optics [10], where the resonator is coupled to the highfrequency Mollow [11] transitions with energy differences $\Delta E \pm \Omega_{R}$. In the present setup the oscillator couples to the much lower Rabi frequency (similar to Ref. [16]), which can be readily tuned to resonance with the oscillator in order to reach the lasing threshold and a proper lasing state.

Laser-like behavior has been predicted also for nanomechanical resonators coupled to superconducting singleelectron transistors [17]. Our proposal differs in two major points: (i) As an active medium we have a coherently driven qubit instead of a dissipative transistor. While transistors usually provide a broad band negative temperature environment for the oscillator, frequencies of order $\omega_{T}$ are resolved in our situation. Experience gained in the field of quantum optics, e.g. from the comparison of Doppler cooling and resolved side-band cooling, shows that frequency resolved regimes lead to stronger effects. (ii) We consider two-photon coupling which allows lasing at the degeneracy point of the qubit where the sensitivity to low-frequency noise is substantially reduced. These facts provide sufficient flexibility in the choice of system parameters and should allow reaching a proper lasing state with photon numbers much above the thermal value and the characteristic line- width narrowing.

We thank E. Il'ichev, K. C. Schwab, and M. D. LaHaye for fruitful discussions. The work is part of the EU IST Project EUROSQIP.

[1] E. Il'ichev et al., Phys. Rev. Lett. 91, 097906 (2003).

[2] A. Wallraff et al., Nature 431, 162 (2004); I. Chiorescu et al., Nature 431, 159 (2004); A. Wallraff et al., Phys. Rev. Lett. 95, 060501 (2005); J. Johansson et al., Phys. Rev. Lett. 96, 127006 (2006);

[3] A. Naik et al., Nature 443, 193 (2006).

[4] O. Buisson et al., Phys. Rev. Lett. 90, 238304 (2003); Yuxi Liu, L. F. Wei, and F. Nori., Europhys. Lett. 67, 941 (2004); I. Martin et al., Phys. Rev. B 69, 125339 (2004); K. Moon and S. M. Girvin, Phys. Rev. Lett. 95, 140504 (2005); M. Mariantoni et al., arXiv:cond-mat/0509737 M. Wallquist, V. S. Shumeiko, and G. Wendin. Phys. Rev. B 74, 224506 (2006); F. Xue et al., New J. Phys. 9, 35 (2007).

[5] A. Blais et al., Phys. Rev. A 69, 062320 (2004).

[6] D. Vion et al., Science 296, 886 (2002).

[7] A. J. Kent et al., Phys. Rev. Lett. 96, 215504 (2006).

[8] A. Yu. Smirnov, Phys. Rev. B 68, 134514 (2003).

[9] Ya. S. Greenberg, E. Il'ichev, and A. Izmalkov, Europhys. Lett. 72, 880 (2005).

[10] J. Zakrzewski, M. Lewenstein, and T.W. Mossberg, Phys. Rev. A 44, 7717 (1991); T.M. Stace, A.C. Doherty, and S.D. Barrett, Phys. Rev. Lett. 95, 106801 (2005).

[11] B. R. Mollow, Phys. Rev. 188, 1969 (1969).

[12] Yi Mu and C. M. Savage, Phys. Rev. A 46, 5944 (1992); J. McKeever et al., Nature 425, 268 (2003).

[13] Ya. S. Greenberg et al., Phys. Rev. B 66, 214525 (2002).

[14] C. W. Gardiner and P. Zoller, Quantum noise (Springer, Heidelberg, 3-d edition, 2004).

[15] M. Reid, K. J. McNeil, and D. F. Walls, Phys. Rev. A 24, 2029 (1981).

[16] D. Jonathan and M. B. Plenio, Phys. Rev. Lett. 87, 127901 (2001).

[17] Ya. M. Blanter, O. Usmani, and Yu. V. Nazarov, Phys. Rev. Lett. 93, 136802 (2004); O. Usmani, Ya. M. Blanter, and Yu V. Nazarov, Phys. Rev. B 75, 195312 (2007); A. A. Clerk and S. Bennett, New J. Phys. 7, 238 (2005); S. D. Bennett and A. A. Clerk, Phys. Rev. B 74, 201301 (2006); M. P. Blencowe, J. Imbers, and A. D. Armour, New J. Phys. 7, 236 (2005); D. A. Rodrigues, J. Imbers, and A. D. Armour, Phys. Rev. Lett. 98, 067204 (2007). 